\title{
Degradación y decoloración de agua contaminada con colorantes textiles mediante procesos de oxidación avanzada
}

\author{
Degradation and decoloration of contaminated water \\ with textile dyes using advanced oxidation processes
}

Laura Castro-Peñal

J. Esteban Durán-Herrera²

Castro-Peña, L:; Durán-Herrera, J.

Degradación y decoloración de agua contaminada con colorantes textiles mediante procesos de oxidación avanzada. Tecnología en Marcha. Vol. 27, N². Pág 40-50 


\section{Palabras clave}

Procesos de oxidación avanzada; tintes textiles; aguas residuales; Taguchi; proceso Foto-Fenton.

\section{Resumen}

En la presente investigación se estudió la efectividad de los procesos de oxidación avanzada $\mathrm{H}_{2} \mathrm{O}_{2} / \mathrm{UV}$ y $\mathrm{H}_{2} \mathrm{O}_{2} / \mathrm{Fe}^{2+} / \mathrm{UV}$ en la degradación y decoloración de agua contaminada con colorante textil COLRON RED SD3B, a manera de contaminante modelo. Para ello se analizó el efecto de la concentración inicial del colorante, el pH de la solución, la concentración de hierro y la concentración de peróxido de hidrógeno mediante un diseño estadístico de experimentos de Taguchi. Del estudio se concluyó que el proceso Foto-Fenton $\left(\mathrm{H}_{2} \mathrm{O}_{2} / \mathrm{Fe}^{2+} / \mathrm{UV}\right)$ genera resultados más favorables en la degradación y decoloración del tinte, en las siguientes condiciones de operación: $\mathrm{pH}=2$, concentraciones de colorante de 100 ppm, de hierro de 80 ppm y de peróxido de hidrógeno de 102 ppm (3,0 mmol/L). Bajo estas condiciones se obtuvieron porcentajes de reducción promedio de $65,5 \%$ en la DQO y de 77,0\% en la decoloración del tinte luego de una hora de tratamiento.

\section{Keywords}

Advanced oxidation processes; textile dye; wastewater; Taguchi; photo-Fenton process.

\begin{abstract}
The degradation and decolorization of azo dye COLRON RED SD3B as model pollutant by $\mathrm{H}_{2} \mathrm{O}_{2}$ / $\mathrm{UV}$ and $\mathrm{H}_{2} \mathrm{O}_{2} / \mathrm{Fe}^{2+} / \mathrm{UV}$ processes were investigated. The effects of dye concentration, solution $\mathrm{pH}$, and $\mathrm{Fe}^{2+}$ and $\mathrm{H}_{2} \mathrm{O}_{2}$ dosages were studied using Taguchi's experimental design method. Photo-Fenton process showed to be more efficient than $\mathrm{H}_{2} \mathrm{O}_{2} / \mathrm{UV}$ process for the color removal and degradation of the dye. The conditions that produced higher degradations rates were $\mathrm{pH}=2$, dye concentration of 100 ppm, ferrous dosage of $80 \mathrm{ppm}$ and hydrogen peroxide dosage of $102 \mathrm{ppm}$ (3.0 mmol/L). Under these conditions, $65.5 \%$ COD reduction and $77.0 \%$ decolorization of the azo dye wastewater were achieved after one hour of treatment.
\end{abstract}

\section{Introducción}

El proceso de elaboración de productos textiles consiste en un gran número de operaciones unitarias que utilizan diversas materias primas como algodón, lana, fibras sintéticas y tintes o colorantes. En la etapa del proceso conocida como de "tintorería y acabados" se generan efluentes líquidos que contienen contaminantes de muy diversa naturaleza, entre ellos sales inorgánicas, almidón, peróxidos, EDTA, tensoactivos, enzimas, surfactantes, colorantes, metales y otros compuestos orgánicos de variada estructura (Gutarra et al., 200I). Una fracción importante de estos contaminantes son los residuos de colorantes textiles. Estos compuestos químicos son poco biodegradables, por lo que presentan gran persistencia en el medio ambiente y los métodos de tratamiento biológicos clásicos no son eficientes en su remoción. Además, la fuerte coloración que imparte a cuerpos de descarga puede llegar a suprimir los procesos fotosintéticos en corrientes de agua, por lo que su presencia debe ser controlada (Marcano, 1990).

Por otro lado, la cada vez más exigente reglamentación en materia de vertidos para su incorporación a las redes de saneamiento o su descarga directa al entorno, obliga a este sector industrial a adecuar la calidad de sus efluentes mediante la implantación de métodos de tratamiento adecuados. La puesta en marcha de procesos de depuración innovadores, eficaces y fiables, acompañados de una creciente política de reciclado de aguas, es una solución que atrae un creciente interés.

En este sentido, el tratamiento mediante Procesos de Oxidación Avanzada (AOPs, siglas en inglés de Advanced Oxidation Processes) de aguas contami- 
nadas con productos orgánicos tóxicos y/o difícilmente biodegradables se perfila como un método adecuado y con grandes posibilidades de desarrollo a corto plazo. Los AOPs consisten en una oxidación química en condiciones de presión y temperatura ambiente, con la cual se puede llegar hasta la mineralización completa de los contaminantes (Kim y Vogelpohl, 2004). Lo anterior viene representado en la siguiente reacción:

$$
\text { AOPs } \rightarrow \cdot \mathrm{OH} \stackrel{\text { Contaminantes }}{\longrightarrow} \mathrm{CO}_{2}+\mathrm{H}_{2} \mathrm{O}+\text { iones inorganicos }
$$

El agente oxidante es un radical denominado hidroxilo $(\bullet \mathrm{OH})$, el cual posee una elevadísima capacidad oxidante, es no selectivo y presenta tiempos de reacción muy cortos (Almecija et al., 20l0). La versatilidad de los AOPs se debe al hecho de que existen diferentes formas de producir los radicales hidroxilo y puede escogerse cual utilizar, dependiendo de los requerimientos específicos de cada agua residual. Entre los métodos más desarrollados e investigados a la fecha están el método de peróxido de hidrógeno y radiación ultravioleta $\left(\mathrm{H}_{2} \mathrm{O}_{2} / U \mathrm{~V}\right)$ y el método conocido como Foto-Fenton $\left(\mathrm{H}_{2} \mathrm{O}_{2} / \mathrm{UV} /\right.$ Fe2+) (López, 200I).

El método $\mathrm{H}_{2} \mathrm{O}_{2} / \mathrm{UV}$ implica la formación de radicales hidroxilo mediante la fotólisis del peróxido de hidrógeno $\left(\mathrm{H}_{2} \mathrm{O}_{2}\right)$ por la acción de la radiación ultravioleta según la siguiente reacción:

$$
\mathrm{H}_{2} \mathrm{O}_{2} \stackrel{U V}{\longrightarrow} 2 \cdot \mathrm{OH}
$$

Las longitudes de onda UV en el rango de 200$280 \mathrm{~nm}$ conducen a la disociación del peróxido de hidrógeno, siendo las lámparas de mercurio con emisión de $254 \mathrm{~nm}$ las más utilizadas (Aleboyeh et al., 2003).

Con relación a la efectividad del proceso $\mathrm{H}_{2} \mathrm{O}_{2} / \mathrm{UV}$, diversos investigadores (Amer et al., 2008; Caronna et al., 1999; Kalt y Galindo, 1999) han llegado a la conclusión de que las variables que producen un efecto significante en el proceso de decoloración y degradación de materia orgánica en el vertido residual de una planta textil son: la concentración de peróxido de hidrógeno $\left(\mathrm{H}_{2} \mathrm{O}_{2}\right)$, el pH, la concentración inicial de colorante y la intensidad de la radiación ultravioleta. Kalt y Galindo (1999) documentaron que el proceso $\mathrm{H}_{2} \mathrm{O}_{2} / \mathrm{UV}$ es más efectivo en un medio ácido $(\mathrm{pH}=3-4)$ en términos de la degradación del color. El hecho de que la eliminación del color en un medio alcalino no sea eficiente se debe a que el peróxido de hidrógeno sufre una descomposición en la cual se forma oxígeno molecular y agua, en lugar de producir radicales hidroxilo; esto hace que la concentración de radicales $\mathrm{OH}^{\circ}$ sea menor a la esperada.

La eficiencia de la decoloración aumenta al incrementarse la dosis de peróxido de hidrógeno hasta un punto en el que alcanza un valor máximo y luego empieza a disminuir. Por ejemplo, en un estudio realizado por Amer et al. (2008) utilizando el método $\mathrm{H}_{2} \mathrm{O}_{2} / \mathrm{UV}$ para el tratamiento de agua residual textil, se determinó que valor máximo de porcentaje de decoloración obtenido fue de $90,69 \%$ con una concentración de $3,9 \% \mathrm{~m} / \mathrm{m}$ de $\mathrm{H}_{2} \mathrm{O}_{2}$ mientras que por encima de esta dosis $(4,8 \% \mathrm{~m} / \mathrm{m})$ este porcentaje se redujo a un $82,3 \%$. Este comportamiento se debe a que cuando existe una concentración alta de $\mathrm{H}_{2} \mathrm{O}_{2}$ ésta va a actuar como un secuestrador de radicales $\mathrm{OH}^{*}$ mientras que a bajas concentraciones de $\mathrm{H}_{2} \mathrm{O}_{2}$ no va a generar los suficientes radicales hidroxilo, que van a ser consumidos por el colorante y consecuentemente baja la tasa de reacción de oxidación (Al-Kdasi et al., 2004).

Hace más de un siglo, H. J. Fenton describió el poder de oxidación del peróxido de hidrógeno en ciertas moléculas orgánicas, bajo la adición de Fe (II) como catalizador (Domènech et al., 2002). Más tarde, se descubrió que esto se debe a la generación de radicales hidroxilo, la cual obedece a la siguiente reacción:

$$
\mathrm{Fe}^{2+}+\mathrm{H}_{2} \mathrm{O}_{2} \rightarrow \mathrm{Fe}^{3+}+\mathrm{OH}^{-}+\cdot \mathrm{OH}
$$

Debido a la simplicidad de la reacción anterior, la reacción de Fenton es el proceso que más se utiliza cuando es necesaria la remoción de compuestos recalcitrantes. Sin embargo, el mayor inconveniente de la reacción de Fenton es la producción de residuos de lodos de hierro. Esto llevó a que se desarrollara el proceso de Foto-Fenton $\left(\mathrm{H}_{2} \mathrm{O}_{2} / \mathrm{UV} / \mathrm{Fe}^{2+}\right)$, el cual utiliza radiación ultravioleta para la reducción del ion Fe (III) a Fe(II), resultando en una drástica reducción de los lodos residuales (Chiu et al., 2007; Vogelpohl, 2007).

Las condiciones de operación en las que se lleva a cabo el proceso Foto-Fenton $(\mathrm{pH}$, la concentración de peróxido de hidrógeno, la temperatura 
y la concentración de aniones inorgánicos), así como la composición del agua residual, afectan sustancialmente la eficacia y velocidad de este proceso de oxidación. En el sistema Foto-Fenton, la velocidad de las reacciones tiende a ser máxima en un pH cercano a 2.8. (Mackay et al., 2006); el valor óptimo se encuentra entre 2,5 y 2,8, donde el Fe(III) existe en solución mayoritariamente como $\mathrm{Fe}(\mathrm{OH})^{2+}\left(\mathrm{H}_{2} \mathrm{O}\right)_{5} \cdot \mathrm{A} \mathrm{pH}$ altos, la velocidad disminuye debido a la precipitación de hierro en forma de $\mathrm{Fe}(\mathrm{OH})_{3}$. La dosis de $\mathrm{H}_{2} \mathrm{O}_{2}$ influye fundamentalmente sobre la eficacia del proceso, mientras que la concentración de hierro afecta la cinética (Baeyens y Neyens, 2003). Tanto la eficacia como la velocidad de reacción tienden a aumentar con la concentración de reactivos. Sin embargo, un exceso de hierro da lugar a una disminución de la eficacia debido a la activación de reacciones secundarias no deseadas, como es el caso de la formación de $\mathrm{Fe}(\mathrm{OH})_{3}$ que puede precipitar, por lo que se ha de tener en cuenta la Kps de esta especie para evitar este problema. Por otro lado, un exceso de peróxido daría lugar a la degradación de este por los radicales hidroxilos, que es una reacción no deseada (Mackay et al., 2006; Domènech et al., 2002).

Diversos investigadores han explorado la eficacia del proceso Foto-Fenton para la degradación de colorantes textiles. Por ejemplo, Khandelwal y Ameta (2013) investigaron la degradación del colorante Basic Yellow 2 utilizando luz visible, obteniendo degradaciones del 100\%. Wu et al. (1999) estudiaron la fotodegradación del colorante Malachite Green en presencia de iones $\mathrm{Fe}^{2+}$ y $\mathrm{Fe}^{3+}$, y compararon la efectividad con respecto a la reacción sin luz. Los resultados demostraron el mejor rendimiento del proceso fotoquímico. La mayoría de la investigaciones se han desarrollado a nivel de reactores batch piloto; muy poca atención se le ha dado a la escala de reactores a nivel industrial en la aplicación de la decoloración de agua residual coloreada. No fue sino hasta la última década que varias investigaciones han reportado el éxito en las aplicaciones de fotoreactores que utilizan el método de oxidación avanzado $\mathrm{H}_{2} \mathrm{O}_{2} / \mathrm{UV}$ para el tratamiento de aguas residuales coloreadas (Chang et al., 2005).

En esta investigación se estudió la efectividad de los procesos de oxidación avanzada $\mathrm{H}_{2} \mathrm{O}_{2} / \mathrm{UV}$ y $\mathrm{Fe}^{2+} /$ $\mathrm{H}_{2} \mathrm{O}_{2} / \mathrm{UV}$ en la decoloración y degradación de agua contaminada con el colorante textil COLRON RED
SD3B, sistema que se utilizó como un agua residual textil modelo. El objetivo principal fue comparar ambas tecnologías y encontrar las condiciones de operación que generaban las mejores eficiencias de degradación del colorante. Para ello se analizó el efecto de la concentración inicial de colorante, el $\mathrm{pH}$ de la solución, la concentración de hierro y la concentración de peróxido de hidrógeno sobre la efectividad de los dos AOPs, empleando un diseño estadístico de experimentos de Taguchi. Este diseño experimental permitió encontrar condiciones de operación adecuadas para llevar a cabo la descontaminación del agua.

\section{Metodología, equipo experimental y materiales}

Todas las pruebas de decoloración y mineralización del colorante COLRON RED SD38 se llevaron a cabo en el sistema mostrado en la figura I. La solución a ser irradiada se alimentaba desde un reservorio de 2,0 L mediante una bomba peristáltica a un flujo continuo de $18,5 \mathrm{~mL} / \mathrm{s}$. Esta disposición permitía tener permanentemente $0,8 \mathrm{I} \mathrm{L}$ de solución en el fotoreactor anular. El fotoreactor (MiniPure MIN- I.5, Atlantic Ultraviolet) comprende una estructura de acero inoxidable $(39,05 \mathrm{~cm}$ largo, 6,35 cm ancho, $7,62 \mathrm{~cm}$ alto), el cual presenta una distancia de 26 $\mathrm{cm}$ aproximadamente entre la entrada y la salida de la solución. En el centro del fotoreactor a lo largo del eje horizontal se encuentra una lámpara ultravioleta de mercurio de baja presión (14 Watts, UV-C, $\lambda \max =254 \mathrm{~nm}$ ), dicha lámpara está protegida por un tubo de cuarzo. Se utilizó un condensador como intercambiador de calor a la salida del reactor para poder mantener la temperatura constante de la solución, este parámetro se controlaba con un termómetro previamente colocado en el reservorio.

El tiempo para cada corrida se fijó en una hora, en intervalos de diez minutos se tomaba del reservorio una alícuota de $25 \mathrm{~mL}$ de la disolución de colorante para realizarle los análisis pertinentes. La concentración de colorante se determinó espectrofotométricamente a $525 \mathrm{~nm}$. La medición de la Demanda Química de Oxígeno se basó en el método estándar establecido por USEPA (Método 410.4). Para ellos se utilizó un colorímetro marca $\mathrm{HACH}$ y un kit de viales del rango de 0 a $150 \mathrm{mg} / \mathrm{L}$ de la marca CHEMETRICS. Para medir la concentración 


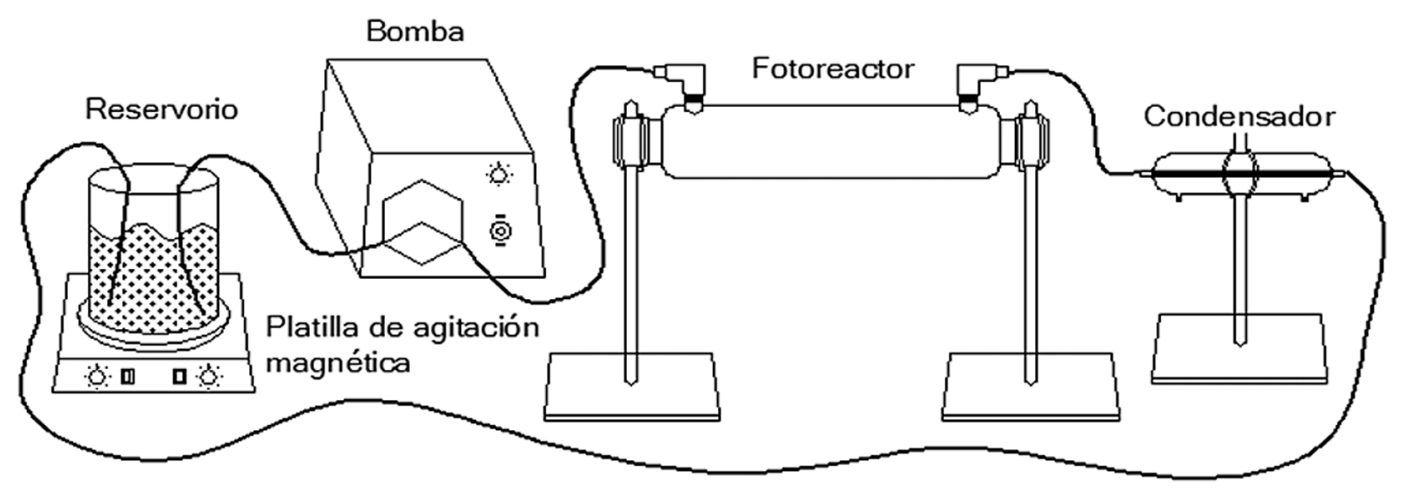

Figura I. Representación esquemática de la configuración del equipo experimental.

del peróxido de hidrógeno $\left(\mathrm{H}_{2} \mathrm{O}_{2}\right)$ en cada muestra, se utilizó un método espectrofotométrico a una longitud de $35 \mathrm{I} \mathrm{nm}$, haciendo reaccionar la muestra con disoluciones de ftalato hidrógeno de potasio $(\mathrm{KHP})$ y yoduro de potasio $(\mathrm{KI})$.

El colorante que se empleó en esta investigación fue el COLRON RED SD3B, cuyo componente reactivo es el C.I. Reactive Red 191 (ver estructura química en la figura 2). Se trabajó con peróxido de hidrógeno al 30\% peso/peso y con sulfato de hierro heptahidratado. El pH de la solución fue ajustado utilizando ácido clorhídrico $(\mathrm{HCl})$ e hidróxido de sodio $(\mathrm{NaOH})$.

Para el análisis estadístico de los resultados se empleó la metodología de Taguchi. En este caso se analizaron cuatro factores a tres niveles cada uno, por lo que se usó un arreglo ortogonal $L_{9}$, que consiste en realizar nueve experimentos para estudiar el efecto de los factores en la degradación y mineralización del colorante. Cada experimento se repitió dos veces con el propósito de cuantificar los errores experimentales. Los cuatro factores analizados fueron: la concentración de colorante, el pH, la concentración de hierro y la concentración de peróxido de hidrógeno $\left(\mathrm{H}_{2} \mathrm{O}_{2}\right)$ según los niveles mostrados en el cuadro I. La metodología de Taguchi indicó cuáles son los niveles de los factores de estudio donde se alcanza un mayor grado de degradación y decoloración del tinte, analizados a través de las variables respuesta: porcentaje de degradación de DQO, porcentaje de decoloración, constante cinética de la reacción de oxidación y relación de gramos de DQO removido por costo de reactivos utilizados.

Para analizar los resultados, el método de Taguchi emplea una medida estadística llamada señal de ruido $(\mathrm{S} / \mathrm{N})$; esta función permite establecer más fácilmente la combinación óptima de parámetros. El<smiles>CS(=O)(=O)CCOS(=O)(=O)Oc1cc(Nc2nc(Cl)nc(Nc3ccc(S(=O)(=O)O)cc3)n2)c2c(O)c(N=Nc3ccc4c([N+](=O)[O-])cccc4c3S(C)(=O)=O)c([N+](=O)[O-])cc2c1</smiles>

Figura 2. Estructura química del C.I. Reactive Red 191.

Cuadro I. Factores y niveles establecidos en la fase experimental de la investigación.

\begin{tabular}{|c|c|c|c|c|}
\hline \multirow{3}{*}{ Nivel } & & & Factores & D \\
\cline { 2 - 5 } & Conc. colorante $(\mathrm{ppm})$ & $\mathrm{B}$ & $\mathrm{C}$ & Conc. $\mathrm{H}_{2} \mathrm{O}_{2}(\mathrm{mmol} / \mathrm{L})$ \\
\hline 1 & 100 & $\mathrm{pH}$ & Conc. Fe(II) (ppm) & 0,3 \\
\hline 2 & 75 & 4,0 & 0 & 1,5 \\
\hline 3 & 50 & 6,0 & 40 & 3,5 \\
\hline
\end{tabular}


cálculo de la señal de ruido es diferente de acuerdo al tipo de característica que se quiera medir. En este caso, las características mayores son mejores, por lo que $\mathrm{S} / \mathrm{N}$ se define como:

$$
S / N=-10 \log \left((1 / n)\left(\sum\left(1 / y^{2}\right)\right)\right)
$$

donde $n$ es el número de datos y y es el valor de cada dato recolectado (Roy, 1990).

\section{Resultados y discusión}

En la figura 3 se observa el efecto de los factores de estudio sobre el porcentaje de degradación del parámetro DQO. Los resultados indican que un incremento en la concentración del colorante disminuye su nivel de degradación. Este resultado es común en los procesos Foto-Fenton y se puede explicar debido a que un incremento en la concentración del tinte puede inducir a un aumento en la densidad óptica interna, es decir, a mayor número de partículas de tinte, la solución se vuelve cada vez más impermeable a la radiación ultravioleta. Por lo tanto, el peróxido de hidrógeno solo va a ser irra-
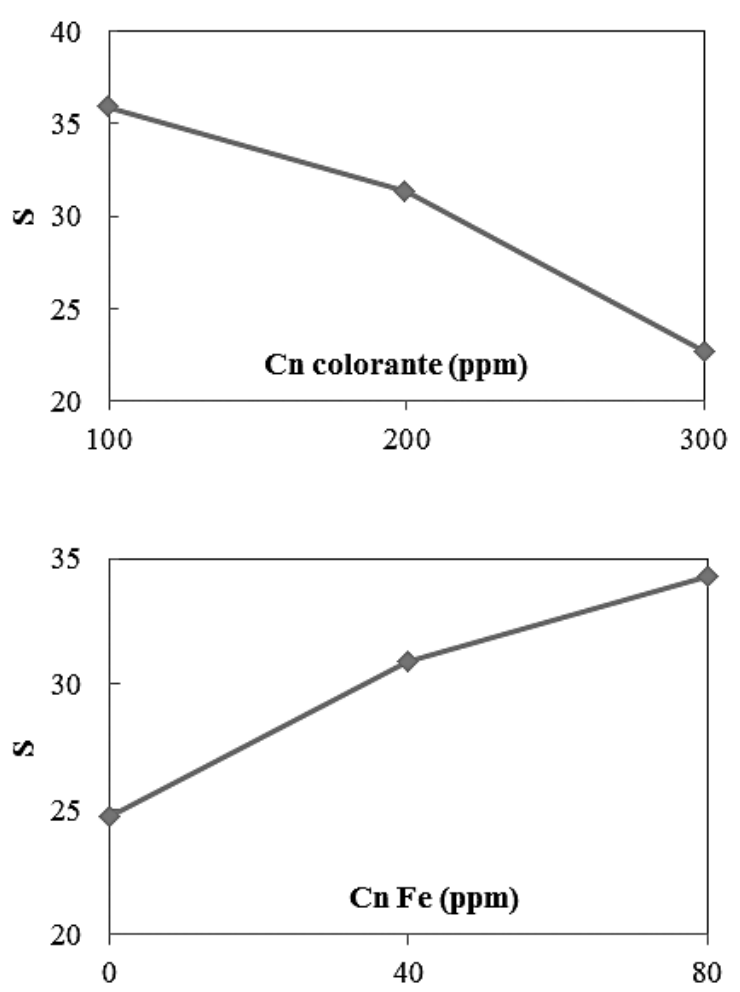

diado por una pequeña porción de luz UV, lo cual hace que la formación de los radicales hidroxilos sea menor y la degradación de la materia orgánica disminuya (Aleboyeh et al., 2005).

Con respecto a las condiciones de $\mathrm{pH}$ estudiadas, los valores de porcentaje de degradación de DQO más altos ocurren a un $\mathrm{pH}$ de 2. Esto se debe a que el $\mathrm{pH}$ controla la producción de radicales hidroxilo libres y la concentración de iones hierro en disolución. Por lo tanto, es un importante parámetro para los procesos Foto-Fenton y $\mathrm{H}_{2} \mathrm{O}_{2} / \mathrm{UV}$. Lo anterior se fundamenta con las reacciones que se dan durante el proceso:

$$
\begin{aligned}
& \mathrm{H}_{2} \mathrm{O}_{2}+\mathrm{Fe}^{2+} \rightarrow \mathrm{Fe}^{3+}+\mathrm{OH}^{-}+\cdot \mathrm{OH} \\
& \mathrm{H}_{2} \mathrm{O}_{2}+\mathrm{UV} \rightarrow 2 \cdot \mathrm{OH} \\
& \mathrm{H}_{2} \mathrm{O}+\mathrm{UV}+\mathrm{Fe}^{3+} \rightarrow \cdot \mathrm{OH}+\mathrm{Fe}^{2+}+\mathrm{OH}^{+}
\end{aligned}
$$

Como se indica en la reacción (5), la cantidad de $\mathrm{OH} \cdot$ que se forma a través del proceso Fenton es afectada por el pH, la formación de $\mathrm{OH}$ • se pueda dar más fácilmente bajo condiciones ácidas. Por el
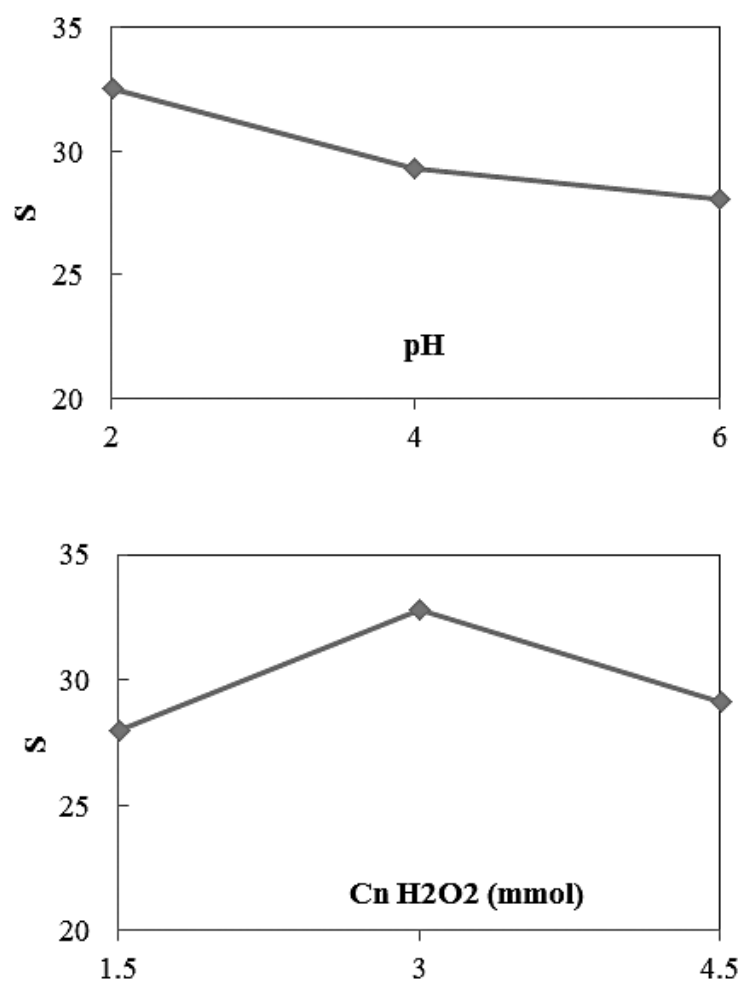

Figura 3. Efectos de los factores de estudio en la señal S/N para el porcentaje de degradación de DQO. 
contrario, se puede notar por la reacción (6) que el proceso $\mathrm{H}_{2} \mathrm{O}_{2} / \mathrm{UV}$ es independiente de este parámetro. Se ha reportado que para el proceso FotoFenton se puede remover de forma efectiva material orgánico tóxico y refractario como es el color de un tinte presente en aguas residuales industriales, a $\mathrm{pH}$ de 3, 3.5 y 7, por lo tanto se concluye que este proceso puede remover contaminantes bajo condiciones ácidas o neutrales. Con base en lo anterior y analizando los resultados de la figura 3, se puede decir que el valor óptimo de $\mathrm{pH}$ para que el proceso Foto-Fenton tenga mayor efectividad es de 2.

Con respecto al efecto de la dosis de peróxido de hidrógeno en la eficiencia de degradación de materia orgánica, se observa que la degradación aumenta cuando se incrementa la dosis de $\mathrm{H}_{2} \mathrm{O}_{2}$ hasta alcanzar un valor máximo; después de este punto el aumento de la concentración de peróxido haría disminuir la eficiencia del proceso. Este comportamiento se debe al hecho de que el peróxido de hidrógeno es un removedor de radicales hidroxilo de acuerdo a las siguientes reacciones:

$$
\begin{aligned}
& \cdot \mathrm{OH}+\mathrm{H}_{2} \mathrm{O}_{2} \rightarrow \mathrm{HO}_{2}^{\cdot}+\mathrm{H}_{2} \mathrm{O} \\
& 2 \mathrm{HO}_{2} \rightarrow \mathrm{H}_{2} \mathrm{O}_{2}+\mathrm{O}_{2} \\
& \cdot \mathrm{OH}+\cdot \mathrm{OH} \rightarrow \mathrm{H}_{2} \mathrm{O}_{2}
\end{aligned}
$$

Cuando existe suficiente peróxido de hidrógeno en la solución, este comienza a competir con el colorante para reaccionar con los radicales hidroxilo ya que $\mathrm{HO}_{2}$ • es menos reactivo que el radical $\mathrm{OH} \bullet$. Por lo tanto, los radicales hidroxilos generados en una alta concentración de ellos van a ser dimerizados a $\mathrm{H}_{2} \mathrm{O}_{2}$. Por lo tanto, es importante aplicar la dosis de peróxido que permita obtener el mayor rendimiento de los procesos $\mathrm{H}_{2} \mathrm{O}_{2} / \mathrm{UV}$ y Foto-Fenton. En esta investigación y de acuerdo con la figura 3, la dosis que permite alcanzar el mayor porcentaje de degradación de DQO es la de $3.0 \mathrm{mmol} / \mathrm{L}$.

Los resultados obtenidos indican que la degradación de la materia orgánica durante el período de reacción inicial fue bastante rápida en la presencia de iones ferrosos, es decir, para el proceso $\mathrm{H}_{2} \mathrm{O}_{2}$ I $\mathrm{Fe}^{2+} / \mathrm{UV}$, mientras esta degradación ocurrió de una manera más lenta cuando no existía hierro en la disolución $\left(\mathrm{H}_{2} \mathrm{O}_{2} / \mathrm{UV}\right)$. Esta tendencia de aumento se debe a que a mayor concentración férrica, mayor es la formación de $\mathrm{OH}$ • que se da en la reacción de Foto-Fenton, lo cual conduce a una mayor tasa de degradación de DQO. Lo anterior se fundamenta con las reacciones (5)-(7).

En la figura 4 se muestra la contribución de los cuatro factores en el porcentaje de degradación de DQO. Se observa que la concentración de colorante es la que presenta el mayor impacto en la variable respuesta. La concentración de hierro es el segundo factor que presenta un mayor impacto porque su presencia o ausencia define el tipo de proceso de oxidación avanzada que se estaría dando y por ende el mecanismo de producción de radicales $\mathrm{OH} \bullet$. El porcentaje de peróxido de hidrógeno y el pH son los factores con las menores contribuciones. Estos resultados fueron además corroborados mediante un análisis de varianza.

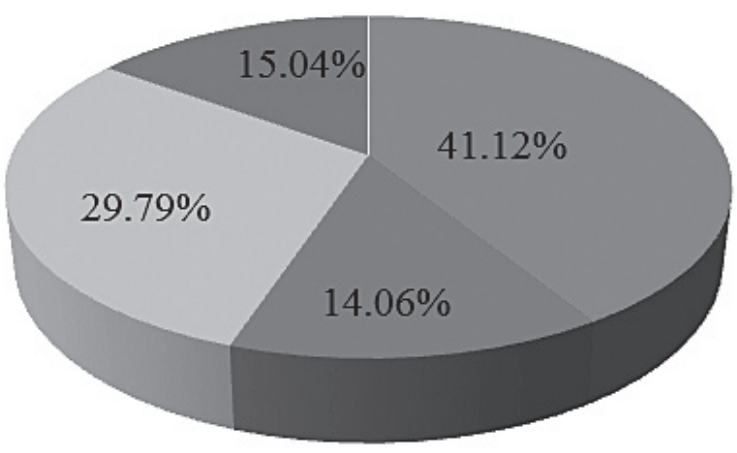

- Cn. Colorante

$\square \mathrm{pH}$

$\square \mathrm{Cn} . \mathrm{Fe}$

$\square \mathrm{Cn} \mathrm{H} 2 \mathrm{O} 2$

Figura 4. Contribución de cada factor de estudio en el porcentaje de degradación de DQO. 
La figura 5 muestra como el grado de decoloración en el proceso Foto-Fenton es mayor y más rápido que en el proceso $\mathrm{H}_{2} \mathrm{O}_{2} / \mathrm{UV}$. Además, a pesar de que la prueba del proceso $\mathrm{H}_{2} \mathrm{O}_{2} / \mathrm{UV}$ presenta el nivel de $\mathrm{H}_{2} \mathrm{O}_{2}$ donde se espera que ocurra un mayor porcentaje de degradación del color, la presencia de hierro es la que domina al final la efectividad del proceso, reiterando que este parámetro presenta una mayor contribución a la degradación del colorante.

Otro punto importante que se quiso estudiar fue el comportamiento que tiene el peróxido de hidrógeno dependiendo del proceso que se está llevando a cabo. Para el proceso Foto-Fenton, el consumo de $\mathrm{H}_{2} \mathrm{O}_{2}$ es decadente durante toda la reacción hasta llegar a su agotamiento, mientras que para proceso $\mathrm{H}_{2} \mathrm{O}_{2} / \mathrm{UV}$, la concentración del peróxido permanece constante durante el tiempo de reacción.

Estos resultados se ilustran en la figura 6 y se pueden explicar debido a que el ion férrico acelera la foto-descomposición del peróxido de hidrógeno, ya que la fotólisis directa de soluciones ácidas de $\mathrm{H}_{2} \mathrm{O}_{2}$ da radicales $\cdot \mathrm{OH}$ como producto primario junto con pequeñas cantidades de radicales $\cdot \mathrm{OOH}$ (ver reacciones (II) y (I2)).

$$
\mathrm{H}_{2} \mathrm{O}_{2}+\mathrm{UV} \rightarrow 2 \mathrm{HO}
$$

$$
\mathrm{H}_{2} \mathrm{O}_{2}+\mathrm{UV} \rightarrow \mathrm{H}^{*}+\mathrm{HOO} .
$$

Para incluir el factor económico en el estudio realizado, el análisis de Taguchi se hizo estudiando la relación de gramos de DQO removidos por dólar de reactivos empleados. Para ello se tomaron en cuenta los costos del peróxido de hidrógeno y del sulfato de hierro, ambos de grado industrial.

En la figura 7 se muestran los resultados obtenidos y se observa que la relación económica de estudio es mayor cuando se trabaja a concentraciones bajas de 100 y 200 ppm; para la concentración de hierro se observa que trabajar a 80 ppm permite obtener una degradación de la materia orgánica favorable incluso cuando el costo de reactivos es mayor; para el caso de la concentración de peróxido de hidrógeno se muestra que a una concentración de entre 1.5 y $3.0 \mathrm{mmol}$ se logra un resultado óptimo. Lo anterior concuerda con lo obtenido en el análisis de la disminución de la DQO.

La contribución de cada factor en la relación de gramos de DQO removidos por dólar invertido viene dada por la figura 8. En ella se observa que las concentraciones de hierro y peróxido de hidrógeno representan las mayores contribuciones a una degradación económica.
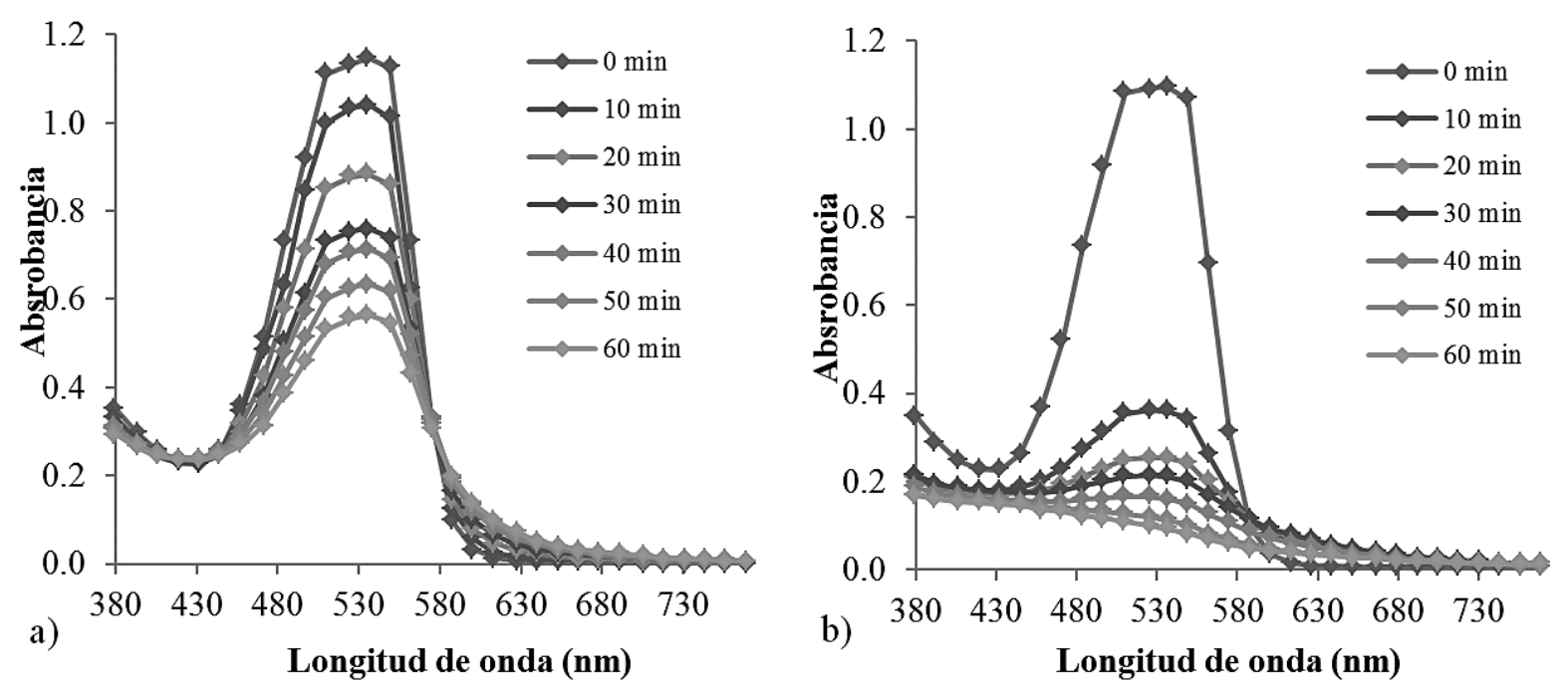

Figura 5. Degradación del color a través del tiempo para una muestra de 300 ppm colorante, a) Proceso H2O2/UV con $3 \mathrm{mmol} / \mathrm{L}$ de $\mathrm{H} 2 \mathrm{O} 2$, b) Proceso Foto-Fenton con $1.5 \mathrm{mmol} / \mathrm{L}$ de $\mathrm{H} 2 \mathrm{O} 2$. 


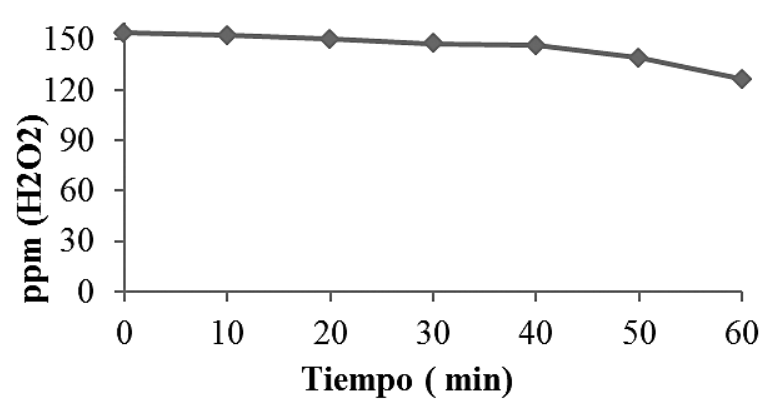

(a)

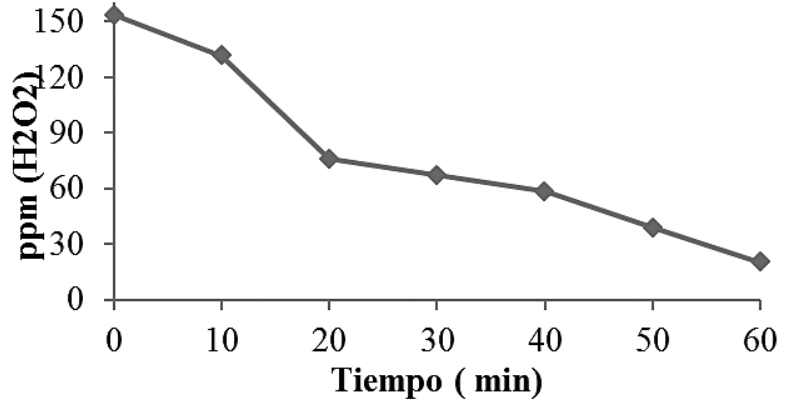

(b)

Figura 6. Comportamiento del peróxido de hidrógeno en el proceso a) H2O2/UV y b) Foto-Fenton.
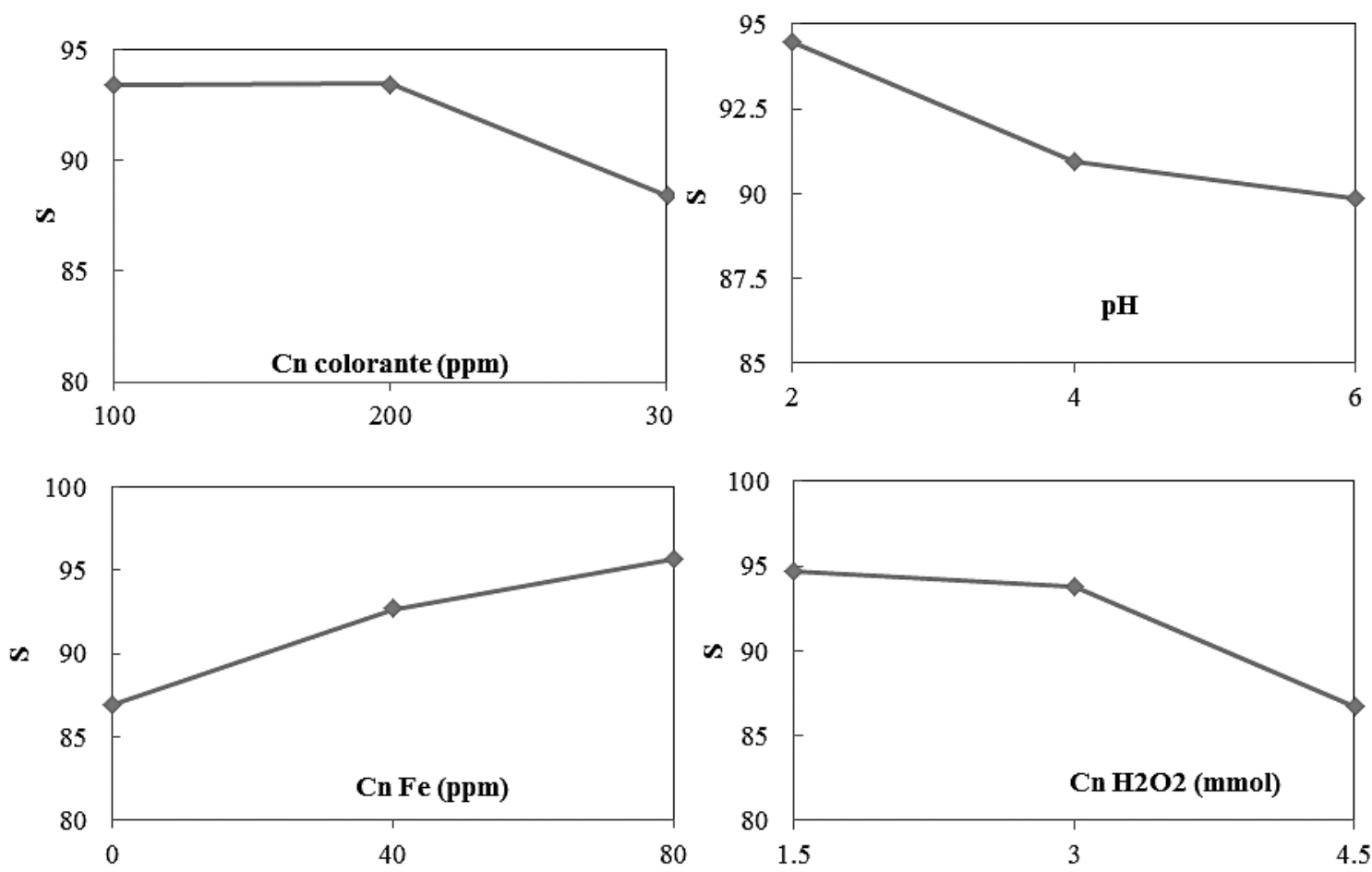

Figura 7. Efectos de los parámetros de diseño en la señal S/N para la relación de gramos de DQO removidos por dólar de costo de reactivos.

Luego de analizar todos estos resultados, se concluyó que las condiciones con las que se alcanzarían mayores y más económicas reducciones de DQO son las que aparecen reportadas en el cuadro 2. De acuerdo a esto, se realizaron dos corridas a las condiciones óptimas encontradas para corroborar los resultados del diseño experimental aplicado (ver figura 9) y se obtuvieron porcentajes de reducción promedio de $65,5 \%$ en la DQO y de $77,0 \%$ en la decoloración del tinte luego de una hora de tratamiento. En la figura 9 se observa el comportamiento típico de reacción del proceso Fenton, en donde se da un decaimiento brusco de la concentración en los primeros minutos de reacción y luego la 


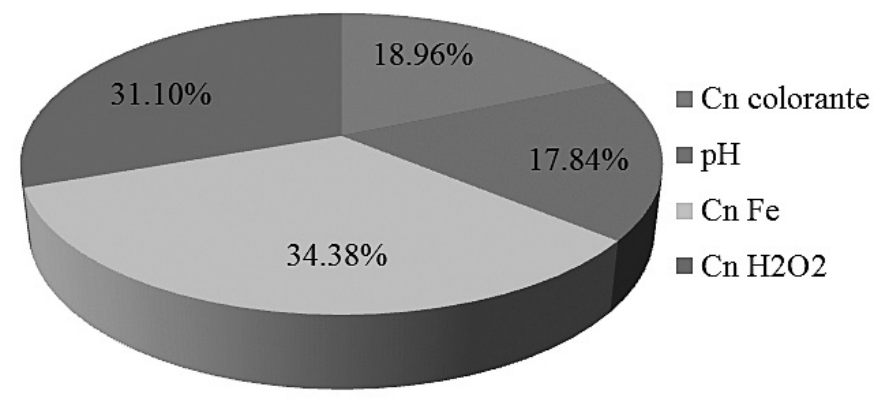

Figura 8. Contribución de cada factor en la relación de gramos de DQO removidos por dólar.

Cuadro 2. Condiciones óptimas de operación para aumentar la efectividad de los procesos en la decoloración y degradación del colorante RRI9I.

\begin{tabular}{|c|c|}
\hline Factor & Valor \\
\hline Conc. de colorante & $100 \mathrm{ppm}$ \\
\hline $\mathrm{pH}$ & 2 \\
\hline Conc. de hierro & $80 \mathrm{ppm}$ \\
\hline Conc. de $\mathrm{H}_{2} \mathrm{O}_{2}$ & $3.0 \mathrm{mmol} / \mathrm{L}$ \\
\hline
\end{tabular}

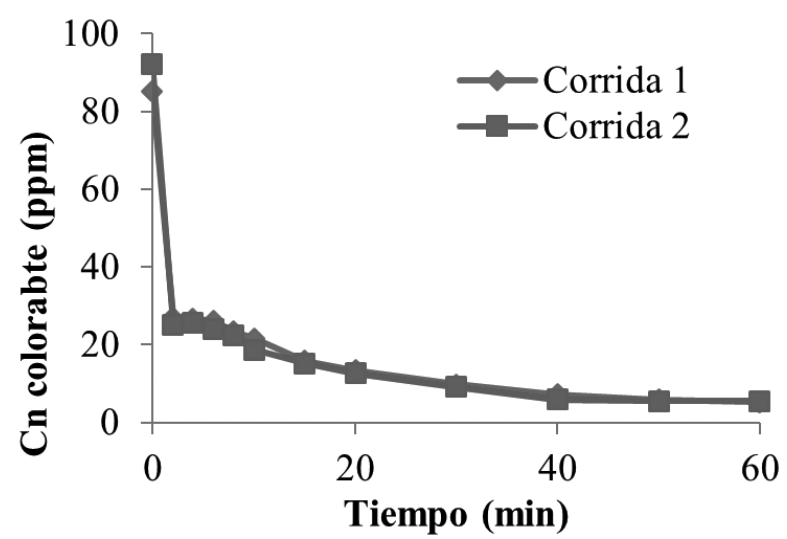

Figura 9. Concentración de colorantes en función del tiempo en las condiciones óptimas

concentración disminuye con una menor velocidad hasta llegar a cero. Esto se debe a que al inicio se da una reacción muy rápida entre los iones $\mathrm{Fe}^{2+}$ con el $\mathrm{H}_{2} \mathrm{O}_{2}$ formando gran cantidad de radicales $\mathrm{OH}$, los cuales reaccionan inmediatamente con el colorante; sin embargo, luego de que los iones se transforman a $\mathrm{Fe}^{3+}$ la velocidad de reacción decae significativamente (Al-Kdasi et al., 2004).

\section{Conclusiones}

Con el desarrollo de esta investigación se encontró que tanto el proceso $\mathrm{H}_{2} \mathrm{O}_{2} / \mathrm{UV}$ como el $\mathrm{H}_{2} \mathrm{O}_{2}$ l $\mathrm{Fe}^{2+} / \mathrm{UV}$ son tratamientos viables para tratar aguas contaminadas con colorante textil, con los que se alcanzaron porcentajes altos en la disminución del color y reducción de su contenido orgánico.

Se concluye que el proceso foto-Fenton presenta una mayor efectividad que el proceso $\mathrm{H}_{2} \mathrm{O}_{2} / \mathrm{UV}$ en las condiciones de estudio, debido a la mayor formación de radicales hidroxilo. La descomposición de $\mathrm{H}_{2} \mathrm{O}_{2}$ catalizada por hierro y UV es mayor que la obtenida solo mediante radiación UV.

La dosis de peróxido de hidrógeno $\left(\mathrm{H}_{2} \mathrm{O}_{2}\right)$ controla la eficiencia global de la remoción de color, mientras que la dosis de hierro juega el rol de acelerar la decoloración durante el período inicial de reacción debido a la formación rápida de los radicales hidroxilo $(\mathrm{OH} \bullet)$. Se encontró que esta remoción de color aumenta con el incremento de la dosis de hierro.

Del análisis de los resultados mediante la metodología Taguchi se obtuvo que las mejores condiciones en las que se alcanzan las mayores y más económicas degradaciones del tinte textil son a concentraciones de colorante de 100 ppm, $\mathrm{pH}$ de 2, concentración de hierro a 80 ppm y concentración de peróxido de hidrógeno de $3.0 \mathrm{mmol} / \mathrm{L}$. 


\section{Recomendaciones}

El trabajo presentado en este artículo no pretende ser un tema finalizado y cerrado sino que, por el contrario, queda abierto para su desarrollo por medio de dos vías complementarias: la investigación básica y la aplicación industrial.

\section{Como investigación básica:}

- Es aconsejable hacer un estudio de las posibles interacciones entre factores, si es que existen, para tener en cuenta todos los fenómenos que contribuyen a la degradación del colorante, haciendo énfasis en una posible interacción del $\mathrm{pH}$ con la concentración de hierro y/o peróxido de hidrógeno.

- Se recomienda realizar un escalamiento piloto para determinar con mayor precisión las influencias de variables operacionales como: temperatura, $\mathrm{pH}$, formación de lodos, tipo de reactor, materiales de soporte.

- Se recomienda el estudio y análisis de los productos intermedios y finales del tratamiento para el estudio de sus características químicas.

Como aplicación, se debe comenzar por trabajar con efluentes reales provenientes de industrias textiles con el objetivo de comprobar:

- La rentabilidad de la aplicación de este método en el tratamiento de aguas residuales.

- La posibilidad de reutilizar los efluentes de tintura tratados con oxidación avanzada.

- El dimensionamiento de la planta de tratamiento.

\section{Bibliografía}

Aleboyeh, A., Aleboyeh, H. \& Moussa, Y. (2003). Critical effect of hydrogen peroxide in photochemical oxidative decolorization of dyes: acid orange 8 , acid blue 74 and methyl orange. Dyes and pigments 57, 67-75.

Aleboyeh, A., Moussa, Y. \& Aleboyeh, H. (2005). The effect of operational parameters on $\mathrm{UV} / \mathrm{H} 2 \mathrm{O} 2$ decolourisation of Acid Blue 74. Dyes and Pigments 66, 129-134.

Al-Kdasi, A., Idris, A., Saed, K. \& Teong, C. (2004). Treatment of textile wastewater by advanced oxidation processes-a review. GLOBAL NEST: the International Journal 6, 222-230.

Almecija, M.C., Hontoria, E., Muñio, M.M., Osorio, F., Poyatos, I.M. \& Torres, I.C. (20।0). Advanced Oxidation Processes for Wastewater Treatment: State of the Art. Water Air Soil Pollution 205, 187-204.

Amer, A., Amin, H., El Fecky, A. \& Ibrahim, I. (2008). Treatment of textile wastewater using $\mathrm{H}_{2} \mathrm{O}_{2} / \mathrm{UV}$ system. Physicochemical Problems of Mineral Processing 42, 17-28.

Baeyens, J. \& Neyens, E. (2003). A review of classic Fenton's peroxidation as an advanced oxidation technique. Journal of Hazardous Materials 98, 33-50

Caronna, T., Colonna, G.M. \& Marcandalli, B. (1999). Oxidative degradation of dyes by ultraviolet radiation in the presence of hydrogen peroxide. Dyes and Pigments 41, 21 I-220.

Chang, M.C. \& Shu, H.Y. (2005). Pilot scale annular plug flow photoreactor by $\mathrm{UV} / \mathrm{H}_{2} \mathrm{O}_{2}$ for the decolorization of azo dye wastewater. Journal of Hazardous Materials 34, 244-25I.

Chiu, H.M., Hung, Y.T., Shiau, C.S. \& Yeh, R.Y. (2007). Degradation and sludge production of textile dyes by Fenton and photoFenton processes. Dyes and Pigments, 73, I-6.

Domènech, X., Peral, J., Pérez, M. \& Torrades, F. (2002). Fenton and photo-Fenton oxidation of textile effluents. Water Research 36, 2703-2710.

Gutarra, A., Lizama, C., Mansilla, H.D. \& Rodríguez, J. (200 I). Tratamiento de residuos líquidos de la industria de celulosa y textil. En: Blesa, M.A. (Ed.). Eliminación de contaminantes por fotocatálisis heterogénea (pp. 285-294). La Plata, Argentina: CYTED.

Kalt, A. \& Galindo, C. (1999). UV/H2O2 oxidation of azodyes in aqueous media: evidence of a structure-degradability relantionship. Dyes and Pigments 42, 199-207.

Khandelwal, D.H. \& Ameta, R. (20/3). Use of Photo-Fenton Reagent in the Degradation of Basic Yellow 2 in Aqueous Medium. Research Journal of Recent Sciences 2, 39-43.

Kim, S.M. \& Vogelpohl, A. (2004). Advanced Oxidation Processes (AOPs) in wastewater Treatment. Journal of Industrial and Engineering Chemistry 10, 33-40.

López, R.C. (200I). Fotodecoloración de un azocolorante mediante el proceso $\mathrm{H}_{2} \mathrm{O}_{2} / \mathrm{UV}$. Tesis presentada para optar por la Licenciatura en Ciencias. Lima: Universidad Nacional de Ingeniería.

Mackay, A., Oliveros, E. \& Pignatello, J.J. (2006). Advanced Oxidation Processes for organic contaminant destruction based on the Fenton reaction and related chemistry. Environmental Science and Technology 36, I-84.

Marcano, D. (1990). Introducción a la química de los colorantes. Caracas: Editorial Reverte.

Roy, R. (1990). A primer on Taguchi Method. New York: Van Nostrand Reinhold Int. Co. Ltd.

Vogelpohl, A. (2007). Applications of AOPs in wastewater treatment. Water Science and Technology 55, 207-2I I.

Wua, K., Xiea, Y., Zhaoa, J. \& Hidakab, H. (1999). Photo-Fenton degradation of a dye under visible light irradiation. Journal of Molecular Catalysis A: Chemical I44, 77-84. 